\title{
Repair of Partial Anomalous Pulmonary Venous Connection: A Rare Case Evaluated by Multi-Slice Computed Tomographic Angiography
}

\author{
Yukun Cao, MD, ${ }^{1}$ Ting Yang, MD,${ }^{2}$ Mai Hou, MD,${ }^{1}$ Hongchao Zhang, $\mathrm{MD},{ }^{1}$ Xia Chen, MD,${ }^{1}$ \\ Fei Zou, $M^{1}$
}

${ }^{1}$ Chinese Air Force General Hospital, Beijing, China; ${ }^{2}$ Chinese PLA General Hospital, Beijing, China

\section{ABSTRACT}

Partial anomalous pulmonary venous connection (PAPVC) is an uncommon congenital heart disease, which may be difficult to identify and often remains undiagnosed. Accurate diagnosis of major aortopulmonary collaterals and partial anomalous pulmonary venous drainage in patients with congenital heart disease is important but problematic. The goal of this publication is to present the diagnosis and surgical repair of this rare pathology in an eight-year-old boy. Atrial septal defect was found by echocardiography, but no anomalous pulmonary vein was found. However, multi-slice computed tomographic angiography (MSCTA) revealed that the isolated right superior pulmonary vein was replaced by right superior pulmonary vein 1 (RSPV1), right superior pulmonary vein 2 (RSPV2) and right superior pulmonary vein 3 (RSPV3), which connected to the superior vena cava (SVC), the orifice of SVC, and the left atrium, respectively. The patient underwent the repair of PAPVC with division of the SVC and re-implantation on the right atrial appendage to restore normal systemic venous drainage. Postoperative course was uneventful. In conclusion, PAPVC is a rare congenital cardiac pathology. MSCTA could contribute to an accurate anatomic and functional definition of this variant.

\section{INTRODUCTION}

Partial anomalous pulmonary venous connection (PAPVC) is an infrequent cause of volume overload of right cardiac chambers and hypervolemia in pulmonary circulation, consisting of the anomalous connection of one or more pulmonary veins to the right atrium or its tributaries [Podzolkov 2016; Sormani P 2016]. Echocardiography plays an important role in the diagnosis of congenital heart disease, but the discovery of vascular anomalies in congenital heart disease remains problematic when using this approach, and the clinical presentation can be varied [Masui 1991]. The utility of multi-slice computed tomographic angiography (MSCTA) permits rapid high-resolution imaging and $3 \mathrm{D}$ resconstruction. It could also provide accurate diagnosis for both optimal management and prognostic evaluation

Received fuly 15, 2017; accepted August 30, 2017.

Correspondence: Yukun Cao, Department of Cardiovascular Center, Chinese Air force General Hospital, 30\# Fucheng Road, Haidian District, Beijing 100142, China. Tel: 86-010-68410099; fax: 86-010-68410037; (e-mail: yexiaotiantg@hotmail.com).
[Gaztanaga 2009]. The anomalous pulmonary veins of this patient were not found by echocardiography but MSCTA. The goal of this publication is to present the diagnosis and surgical repair of this uncommon variant.

\section{CASE REPORT}

An eight-year-old boy was admitted to our cardiovascular center for an evaluation of exertional dyspnea. Upon physical examination, a grade II systolic murmur was detected. Echocardiography showed right atrium and ventricular enlargement. An atrial septal defect (ASD) and a mild tricuspid regurgitation with $\mathrm{PAP}$ of $33 \mathrm{~mm} \mathrm{Hg}$ were revealed (Figure 1). The global left ventricle (LV) ejection fraction was $55 \%$. However, the anomalous pulmonary veins of this patient were not found by echocardiography. Preoperative MSCTA revealed that the isolated right superior pulmonary vein was replaced by right superior pulmonary vein one (RSPV1), right superior pulmonary vein two (RSPV2) and right superior pulmonary vein three (RSPV3), which connected to the superior vena cava (SVC) about $2.5 \mathrm{~cm}$ above the atriocaval junction, the orifice of SVC (atrio-caval junction), and the left atrium respectively (Figure 2).

Surgical repair of the anomalous veins and defect were performed with cardiopulmonary bypass via median sternotomy. Intraoperative examination confirmed the preoperative evaluation by MSCTA. We did not find the normal structure of the right superior pulmonary vein connected to the left atrium; on the contrary, there were three anomalous pulmonary veins (RSPV1, RSPV2 and RSPV3) connected to the SVC, the orifice of SVC, and the left atrium respectively (Figure 3).

The ASD was enlarged to ensure unobstructed drainage of the pulmonary veins to the left atrium. A synthetic patch was sutured to the anterior margin of the ASD and around the orifice of the SVC, thus baffling the proximal end of SVC and pulmonary venous drainage to the left atrium. The SVC was divided above the anomalous pulmonary veins and oversewn proximally. The repair was completed by anastomosing the

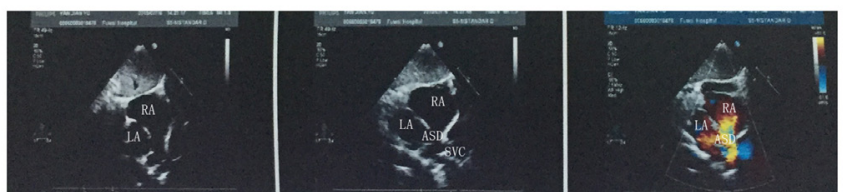

Figure 1. Preoperative Echocardiography: subcostal two chamber view. LA indicates left atrium; RA, right atrium; SVC, superior vena cava; ASD, atrial septal defect. 

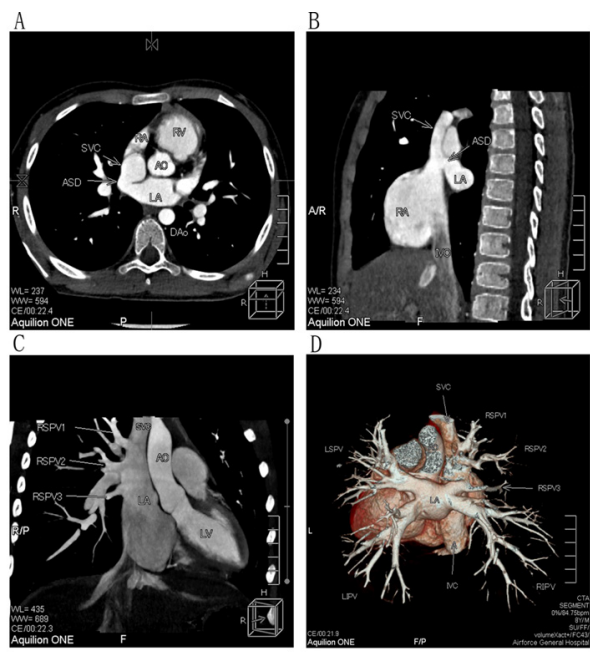

Figure 2. Preoperative multi-slice computed tomographic angiography. A, axial plane image; $B$, in left anterior oblique positon; $C$, in right anterior oblique positon; $\mathrm{D}$, posterior view of $3 \mathrm{D}$ construction. Ao indicates aorta; LA, left atrium; LV, left ventricle; RA right atrium; RV, right ventricle; SVC, superior vena cava; ASD, atrial septal defect; RIPV, right inferior pulmonary vein; RSPV1, right superior pulmonary vein 1; RSPV2, right superior pulmonary vein 2; RSPV3, right superior pulmonary vein 3; LIPV, left inferior pulmonary vein; LSPV, left superior pulmonary vein.

distal end of the SVC to the open right atrial appendage, which helped to restore normal systemic SVC drainage. It is very important to reduce the risk of SVC obstruction (Figure 3).

Postoperative course was uneventful. Mechanical ventilation was discontinued after five hours. The patient was discharged from the clinic in good condition on the seventh postoperative day.

\section{DISCUSSION}

PAPVC can be difficult to identify and often remains undiagnosed. Substantial anatomic variability exists among patients with PAPVC. It is not characterized by specific signs and symptoms, and could mimic other causes of right chamber enlargement or hypervolemia in pulmonary circulation. A variety of techniques have been suggested for correction of PAPVC [Kyger 1978; Chartrand 1976]. However, surgical repair of PAPVC can be complicated by obstruction of the SVC, obstruction of the pulmonary veins, and the postoperative arrhythmias [Trusler 1980]. Accurate preoperative evaluation is necessary, as it plays an important role in decision making for surgery. Early detection and treatment are associated with a better outcome but require accurate demonstration of the anatomic distribution of the anomalous vessels.

We present a rare case of PAPVC in which the normal isolated right superior pulmonary vein was replaced by three anomalous pulmonary veins that connected to the SVC, the orifice of SVC, and the left atrium, respectively. Echocardiography is the first line diagnostic tool in patients with suspect right chambers enlargement but it may provide limited information, as occurred in our case. MSCTA is a reliable

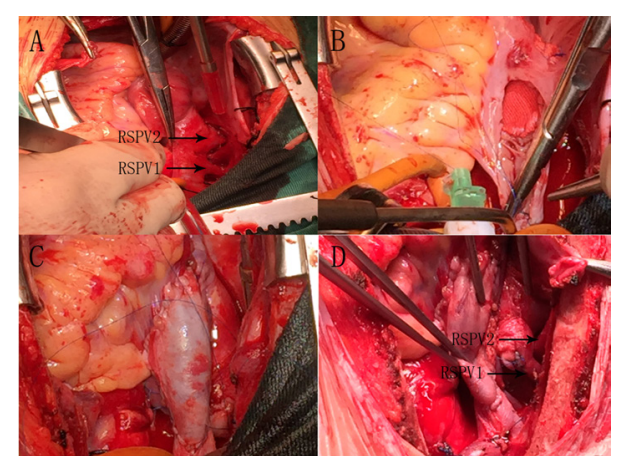

Figure 3. Surgical repair of the anomalous veins and defect. A, intraoperative examination; $B$, suture a synthetic patch to the anterior margin of the ASD and around the orifice of the SVC; C, anastomose the distal end of the SVC to the open right atrial appendage; D, operation completed. RSPV1 indicates right superior pulmonary vein 1; RSPV2, right superior pulmonary vein 2 .

diagnostic method providing a noninvasive assessment of three-dimensional anatomy with excellent spatial resolution. Its ability to obtain visualization of the heart, vessels and surrounding organs is very helpful for us to choose optimal surgical tactics. In this case, the high insertion (about $2.5 \mathrm{~cm}$ above the atriocaval junction) of RSPV1 added to the complexity of the repair. We also needed to repair RSPV2 at the same time. Combined with the preoperative evaluation through MSCTA, we finally decided to repair PAPVC with division of the SVC and reimplantation on the right atrial appendage to restore normal systemic venous drainage. Postoperative course was uneventful and no complication such as obstruction occurred.

In conclusion, PAPVC is a rare congenital cardiac structural abnormality, of which MSCTA could contribute to the precise and timely diagnosis. This is consequential, as the accurate preoperative evaluation is key to the success of operation.

\section{REFERENCES}

Chartrand C, Payot M, Davignon A, et al. 1976. A new surgical approach for correction of partial anomalous pulmonary venous drainage into the superior vena cava. J Thorac Cardiovasc Surg 71:29-34.

Gaztanaga J, Pizarro G, Sanz J. 2009. Evaluation of cardiac valves using multidetector CT. Cardiol Clin 27:633-44.

Kyger ER II, Frazier OH, Cooley DA, et al. 1978. Sinus venosus atrial septal defect: early and late results following closure in 109 patients. Ann Thorac Surg 25:44-50.

Masui T, Seelos KC, Kersting-Sommerhoff BA, et al. 1991. Abnormalities of the pulmonary veins: evaluation with MR imaging and comparison with cardiac angiography and echocardiography. Radiology 181:645-649.

Podzolkov VP, Chiaureli MR, Kovalev DV, et al. 2016. Repair of isolated partial anomalous pulmonary venous connection of the right upper pulmonary veins to the left vertical vein. Interact CardioVasc Thorac Surg 23:501-2.

Sormani P, Roghi A, Cereda A, et al. 2016. Partial Anomalous Pulmonary Venous Return as Rare Cause of Right Ventricular Dilation: A Retrospective Analysis. Congenit Heart Dis 11:365-8.

Trusler GA, Kazenelson G, Freedom RM, et al. 1980. Late results following repair of partial anomalous pulmonary, venous connection with sinus venosus atrial septal defect. J Thorac Cardiovasc Surg 79:776-81. 